\title{
A Feature Selection based Ensemble Classification Framework for Software Defect Prediction
}

\author{
Ahmed Iqbal, Shabib Aftab, Israr Ullah, Muhammad Salman Bashir, Muhammad Anwaar Saeed \\ Department of Computer Science, Virtual University of Pakistan \\ Email: ahmedeqbal@gmail.com, shabib.aftab@gmail.com, israr.ullah@vu.edu.pk, salman.vu@gmail.com, \\ anwaar@vu.edu.pk
}

Received: 26 July 2019; Accepted: 23 August 2019; Published: 08 September 2019

\begin{abstract}
Software defect prediction is one of the emerging research areas of software engineering. The prediction of defects at early stage of development process can produce high quality software at lower cost. This research contributes by presenting a feature selection based ensemble classification framework which consists of four stages: 1) Dataset selection, 2) Feature Selection, 3) Classification, and 4) Results. The proposed framework is implemented from two dimensions, one with feature selection and second without feature selection. The performance is evaluated through various measures including: Precision, Recall, F-measure, Accuracy, MCC and ROC. 12 Cleaned publically available NASA datasets are used for experiments. The results of both the dimensions of proposed framework are compared with the other widely used classification techniques such as: "Naïve Bayes (NB), Multi-Layer Perceptron (MLP). Radial Basis Function (RBF), Support Vector Machine (SVM), K Nearest Neighbor (KNN), $\mathrm{kStar}\left(\mathrm{K}^{*}\right)$, One Rule (OneR), PART, Decision Tree (DT), and Random Forest (RF)". Results reflect that the proposed framework outperformed other classification techniques in some of the used datasets however class imbalance issue could not be fully resolved.
\end{abstract}

Index Terms - Ensemble Classifier, Hybrid Classifier, Random Forest, Software Defect Prediction, Feature Selection

\section{INTRODUCTION}

Today, the production of high quality software at lower cost is challenging due to the large size and high complexity of required systems [1,2], [23]. However this issue can be resolved if we can predict about the particular software modules in advance, where defects are more likely to occur in future [3], [10]. The process of predicting a defective module is known as software defect prediction in which we predict the future defects at the early stages of software development life cycle (before the testing). It is considered as one of the challenging tasks of quality assurance process. Identification of defective modules at the early stage is vital as the cost of correction increases at later stages of development life cycle. Software metrics extracted from historical software data is used to predict the defective modules [29,30,31,32]. Machine learning techniques have been proved as a promising way for effective and efficient software defect prediction. These techniques are categories as 1) supervised, 2) un-supervised, and 3) hybrid. The supervised technique needs a pre-classified (training data) in order to train the classifier. During training the rules are developed which are further used to classify the unseen data (test data). In unsupervised techniques no training data is needed as these techniques use particular algorithm to identify the classes and maintain. The hybrid approach integrates the both (supervised and un-supervised). This paper proposed a feature selection based ensemble classification framework for software defect prediction. The framework is implemented from two dimensions, one with the feature selection and second without the feature selection, so that the difference of results in both dimensions can be analyzed and discussed. Each dimension further used two techniques Bagging and Boosting with Random Forest. Performance evaluation is performed from various measures such as: Precision, Recall, F-measure, Accuracy, MCC and ROC. Clean version of 12 publically available NASA datasets are used in this research including: "CM1, JM1, KC1, KC3, $\mathrm{MC} 1, \mathrm{MC} 2, \mathrm{MW} 1, \mathrm{PC} 1, \mathrm{PC} 2, \mathrm{PC} 3, \mathrm{PC} 4$ and PC5". The results of the proposed framework are also compared with other widely used supervised classification techniques such as: "Naïve Bayes (NB), Multi-Layer Perceptron (MLP). Radial Basis Function (RBF), Support Vector Machine (SVM), K Nearest Neighbor (KNN), $\mathrm{kStar}\left(\mathrm{K}^{*}\right)$, One Rule (OneR), PART, Decision Tree (DT), and Random Forest (RF)". According to results the proposed framework showed higher performance in some of the used datasets but the class imbalance problem is not fully resolved. The class imbalance issue in software defect datasets is one of the main reason of lower and biased performance of classifiers [22,23]. 


\section{RELATED WORK}

Many researchers have used machine learning techniques to resolve the classification problems in various areas including: sentiment analysis $[11,12,13,14,15,16]$, network intrusion detection [17] "in press"[18],[19], rainfall prediction [20,21], and software defect prediction [10], [29] etc.. Some selected studies regarding the software defect predictions are discussed here briefly. In [10] the researchers compared the performance of various supervised machine learning techniques on software defect prediction and used 12 NASA datasets for experiments. The authors have highlighted that Accuracy and ROC did not show any reaction on class imbalance issue however Precision, Recall, F-Measure and MCC reacted on this issue with a symbol of "?" in results. In [24], the researchers used six classification techniques for software defect prediction and used the data of 27 academic projects for experiment. The used techniques are: Discriminant Analysis, Principal Component Analysis (PCA), Logistic Regression (LR), Logical Classification, Holographic Networks, and Layered Neural Networks model. Backpropagation learning technique was used to train ANN. Performance evaluation was performed by using following measures: Verification Cost, Predictive Validity, Achieved Quality and Misclassification Rate. The results reflected that, no classification technique performed better on software defect prediction in the experiment. In [25] the researchers predicted the software defects by using SVM and compared the performance with other widely used prediction techniques including: Logistic Regression (LR), K-Nearest Neighbors (KNN), Decision Trees, Multilayer Perceptron (MLP), Bayesian Belief Networks (BBN), Radial Basis Function (RBF), Random Forest (RF), and Naïve Bayes, (NB). For experiments, NASA datasets are used including: PC1, $\mathrm{CM} 1, \mathrm{KC} 1$ and $\mathrm{KC} 3$. According to results SVM outperformed some of the other classification techniques. In [26] the researchers explored and discussed the significance of particular software metrics for the prediction of software defects. They identified the significant software metrics with the help of ANN after training with historical data. After that the extracted and shortlisted metrics were used to predict the software defects through another ANN model. The performance of the proposed technique was compared with Gaussian kernel SVM. JM1 dataset from NASA MDP repository was used for experiment. According to results the SVM performed better than ANN in binary defect classification. Researchers in [27] proposed a technique for software defect prediction which includes a novel Artificial Bee Colony (ABC) algorithm with Artificial Neural Network in order to find the optimal weights. For experiment, five publically available datasets were used from NASA MDP repository and the results reflected the higher results of proposed technique as compared to other classification techniques. In [28], the researchers introduced an approach which consists of Hybrid Genetic algorithm and Deep Neural Network. Hybrid Genetic algorithm is used for the selection and optimization of features whereas Deep Neural Network is used for classification by focusing on the selected features. The experiments were carried out on the PROMISE datasets and the results showed the higher performance of proposed approach as compared to other defect prediction techniques.

\section{MATERIALS AND METHODS}

This research proposes a feature selection based ensemble classification framework to predict the software defects.

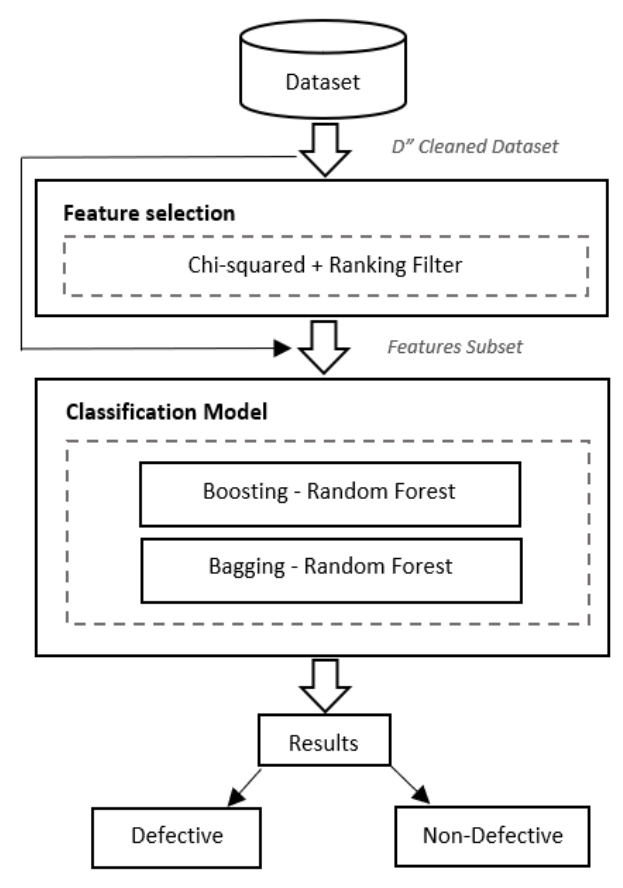

Fig.1. Proposed Classification Framework.

The proposed framework (Fig. 1) consists of four stages: 1) Dataset selection, 2) Feature Selection, 3) Classification, and 4) Results. The framework is implemented in two dimensions, in first, the feature selection stage is skipped and datasets are directly given to the ensemble classifiers however in second dimension the datasets gone through the feature selection stage. The performance of both the dimensions of proposed framework is compared with other widely used classifiers such as: "Naïve Bayes (NB), Multi-Layer Perceptron (MLP), Radial Basis Function (RBF), Support Vector Machine (SVM), K Nearest Neighbor $(\mathrm{kNN}), \mathrm{kStar}\left(\mathrm{K}^{*}\right)$, One Rule (OneR), PART, Decision Tree (DT), and Random Forest (RF)". All the experiments are performed in WEKA [5], which is the widely used data mining tools. It is developed in Java language at the University of Waikato, New Zealand. It is widely accepted due to its portability, General Public License and ease of use.

Dataset selection is the first stage of proposed framework. Twelve publically available cleaned NASA datasets are used in this research for experiment. The datasets include: "CM1, JM1, KC1, KC3, MC1, MC2, MW1, PC1, PC2, PC3, PC4 and PC5 (Table 2)". Each 
dataset belongs to a particular NASA's software system, and consists of various quality metrics in the form of attributes along with known output class. The output class is also known as target class and is predicted on the basis of other available attributes. The target/output class is known as dependent attribute whereas other attributes which are used to predict the dependent attribute are known as independent attributes. The datasets used in this research included dependent attribute having values either "Y" or "N". "Y" reflects that the particular instance (module) is defective and " $\mathrm{N}$ " means it is nondefective. The researchers in [4] provided two versions of clean datasets: DS' ("which included duplicate and inconsistent instances") and D" ("which do not include duplicate and inconsistent instances"). Table 1 reflects the cleaning criteria implemented by [4]. We have used D' (Table 2) version in this research which is taken from [6]. These cleaned datasets are already used and discussed by $[7,8,9,10]$.

Table 1. Cleaning Criteria [4]

\begin{tabular}{|l|l|l|}
\hline Criterion & $\begin{array}{l}\text { Data Quality } \\
\text { Category }\end{array}$ & Explanation \\
\hline 1. & Identical cases & $\begin{array}{l}\text { "Instances that have identical values } \\
\text { for all metrics including class label". }\end{array}$ \\
\hline 2. & $\begin{array}{l}\text { Inconsistent } \\
\text { cases }\end{array}$ & $\begin{array}{l}\text { "Instances that satisfy all conditions } \\
\text { of Case 1, but where class labels } \\
\text { differ". }\end{array}$ \\
\hline 3. & $\begin{array}{l}\text { Cases with } \\
\text { missing values } \\
\text { "Instances that contain one or more } \\
\text { missing observations". }\end{array}$ \\
\hline 4. & $\begin{array}{l}\text { Conflicting } \\
\text { feature values }\end{array}$ & $\begin{array}{l}\text { "Instances that have 2 or more } \\
\text { metric values that violate some } \\
\text { referential integrity constraint. For } \\
\text { example, } \\
\text { LOC TOTAL is less than } \\
\text { Commented LOC. Hower, } \\
\text { Commented LOC is a subset of LOC } \\
\text { TOTAL". }\end{array}$ \\
\hline 5. & $\begin{array}{l}\text { "Instances that violate some integrity } \\
\text { constraint. For example, value of } \\
\text { LOC=1.1". }\end{array}$ \\
\hline
\end{tabular}

Table 2. NASA Cleaned Datasets D', [4], [7]

\begin{tabular}{|c|c|c|c|c|c|}
\hline Dataset & Attributes & Modules & Defective & $\begin{array}{c}\text { Non- } \\
\text { Defective }\end{array}$ & $\begin{array}{c}\text { Defective } \\
(\%)\end{array}$ \\
\hline CM1 & 38 & 327 & 42 & 285 & 12.8 \\
\hline JM1 & 22 & 7,720 & 1,612 & 6,108 & 20.8 \\
\hline KC1 & 22 & 1,162 & 294 & 868 & 25.3 \\
\hline KC3 & 40 & 194 & 36 & 158 & 18.5 \\
\hline MC1 & 39 & 1952 & 36 & 1916 & 1.8 \\
\hline MC2 & 40 & 124 & 44 & 80 & 35.4 \\
\hline MW1 & 38 & 250 & 25 & 225 & 10 \\
\hline PC1 & 38 & 679 & 55 & 624 & 8.1 \\
\hline PC2 & 37 & 722 & 16 & 706 & 2.2 \\
\hline PC3 & 38 & 1,053 & 130 & 923 & 12.3 \\
\hline PC4 & 38 & 1,270 & 176 & 1094 & 13.8 \\
\hline PC5 & 39 & 1694 & 458 & 1236 & 27.0 \\
\hline
\end{tabular}

Feature selection is the second and the most significant stage of proposed classification framework. This stage selects the optimum set of features for effective classification results. Many researchers have reported that most of the datasets only have few of the independent features which can predict the target class effectively whereas remaining features do not participate well and even can reduce the performance of classifier if not removed. We have used Chi-Square as attribute evaluator along with Ranker search method as feature selection technique.

Third stage deals with the classification with ensemble classifiers. Besides the feature selection, ensemble learning techniques have also been reported as an efficient way to improve the classification results. Bagging and Boosting are the two widely used ensemble techniques provided by Weka, which are also known as meta-learners. These techniques work by taking the base learner as argument and create a new learning algorithm by manipulating the training data. We have used Bagging and Boosting along with Random Forest as base classifier in the proposed framework.

Finally the fourth (result) stage reflects the classified modules along with the accuracy of proposed framework. The results are analyzed and discussed in detail in the next section.

\section{RESULTS AND DISCUSSION}

This section reflects the performance of proposed framework. The performance evaluation is performed in terms of various measures generated from confusion matrix (Fig. 2).

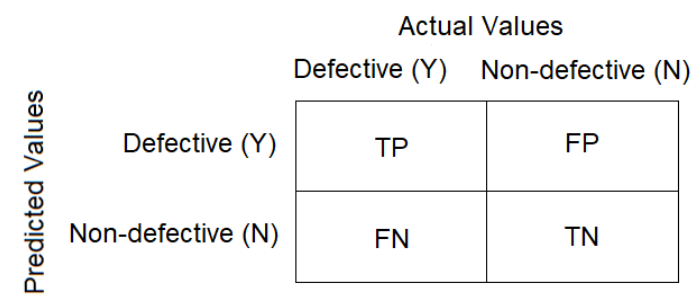

Fig.2. Confusion Matrix.

A confusion matrix consists of the following parameters:

True Positive (TP): "Instances which are actually positive and also classified as positive".

False Positive (FP): "Instances which are actually negative but classified as positive".

False Negative (FN): "Instances which are actually positive but classified as negative".

True Negative (TN): "Instances which are actually negative and also classified as negative".

The performance of both the dimensions of proposed framework is evaluated through following measures: Precision, Recall, F-measure, Accuracy, MCC and ROC [22]. These measures are calculated from the parameters of confusion matrix as shown below. 


$$
\begin{aligned}
& \text { Precision }=\frac{T P}{(T P+F P)} \\
& \operatorname{Recall}=\frac{T P}{(T P+F N)}
\end{aligned}
$$

$$
A U C=\frac{1+T P_{r}-F P_{r}}{2}
$$

$$
M C C=\frac{T N * T P-F N * F P}{\sqrt{(F P+T P)(F N+T P)(T N+F P)(T N+F N)}}
$$

The proposed framework classified the datasets in two dimensions 1) with feature selection and 2) without feature selection. In each dimension the Random Forest classifier is used with Bagging and Boosting techniques so there are total of four techniques in the proposed framework 1) Bagging-RF, 2) Boosting-RF, Feature Selection-Bagging-RF, 4) Feature-Selection-Boosting-RF. Each of the table which reflects the results also shows the score of other classification techniques such as: "Naïve Bayes (NB), Multi-Layer Perceptron (MLP). Radial Basis Function (RBF), Support Vector Machine (SVM), $\mathrm{K}$ Nearest Neighbor (KNN), kStar $\left(\mathrm{K}^{*}\right)$, One Rule (OneR), PART, Decision Tree (DT), and Random Forest (RF)". These results are taken from a published paper [10] in order to compare the performance of proposed framework. The paper [10] have used the same datasets (D') for experiments.

The results of Precision, Recall and F-Measure of each dataset for each class ( $\mathrm{Y}$ and $\mathrm{N}$ ) are reflected in the tables from Table 3 to Table 14. Highest scores in each class are

\begin{tabular}{|c|c|c|c|c|}
\hline & $\mathrm{N}$ & 0.9070 & 0.9890 & 0.9460 \\
\hline \multirow[t]{2}{*}{ Boost-RF } & $\mathrm{Y}$ & 0.0000 & 0.0000 & 0.0000 \\
\hline & $\mathrm{N}$ & 0.9070 & 0.9890 & 0.9460 \\
\hline \multirow[t]{2}{*}{ Bag-RF } & $\mathrm{Y}$ & 0.0000 & 0.0000 & 0.0000 \\
\hline & $\mathrm{N}$ & 0.9070 & 0.9890 & 0.9460 \\
\hline \multirow{2}{*}{$\begin{array}{l}\text { Boost-RF- } \\
\text { FS }\end{array}$} & $\mathrm{Y}$ & 0.0000 & 0.0000 & 0.0000 \\
\hline & $\mathrm{N}$ & 0.9070 & 0.9890 & 0.9460 \\
\hline \multirow{2}{*}{$\begin{array}{l}\text { Bag-RF- } \\
\text { FS }\end{array}$} & $\mathrm{Y}$ & 0.0000 & 0.0000 & 0.0000 \\
\hline & $\mathrm{N}$ & 0.9070 & 0.9890 & 0.9460 \\
\hline
\end{tabular}
highlighted in bold for easy identification.

Table 3. CM1 Data Results

\begin{tabular}{|c|c|c|c|c|}
\hline Classifier & Class & Precision & Recall & F-Measure \\
\hline \multirow{2}{*}{ NB } & $\mathrm{Y}$ & $\mathbf{0 . 1 6 7 0}$ & $\mathbf{0 . 2 2 2 0}$ & $\mathbf{0 . 1 9 0 0}$ \\
\cline { 2 - 5 } & $\mathrm{N}$ & $\mathbf{0 . 9 1 9 0}$ & 0.8880 & 0.9030 \\
\hline \multirow{3}{*}{ RLP } & $\mathrm{Y}$ & 0.0000 & 0.0000 & 0.0000 \\
\cline { 2 - 5 } & $\mathrm{N}$ & 0.9040 & 0.9550 & 0.9290 \\
\hline \multirow{3}{*}{ SVM } & $\mathrm{Y}$ & $?$ & 0.0000 & $?$ \\
\cline { 2 - 5 } & $\mathrm{N}$ & 0.9080 & $\mathbf{1 . 0 0 0 0}$ & $\mathbf{0 . 9 5 2 0}$ \\
\cline { 2 - 5 } & $\mathrm{Y}$ & $?$ & 0.0000 & $?$ \\
\hline \multirow{3}{*}{ kNN } & $\mathrm{N}$ & 0.9080 & $\mathbf{1 . 0 0 0 0}$ & $\mathbf{0 . 9 5 2 0}$ \\
\cline { 2 - 5 } & $\mathrm{Y}$ & 0.0670 & 0.1110 & 0.0830 \\
\hline \multirow{2}{*}{ kStar } & $\mathrm{Y}$ & 0.9040 & 0.8430 & 0.8720 \\
\cline { 2 - 5 } & $\mathrm{N}$ & 0.0670 & 0.1110 & 0.0830 \\
\hline \multirow{2}{*}{ PART } & $\mathrm{Y}$ & 0.0000 & 0.0000 & 0.0000 \\
\cline { 2 - 5 } & $\mathrm{N}$ & 0.9030 & 0.9440 & 0.9230 \\
\hline \multirow{2}{*}{ DT } & $\mathrm{Y}$ & $?$ & 0.0000 & $?$ \\
\cline { 2 - 5 } & $\mathrm{N}$ & 0.9080 & $\mathbf{1 . 0 0 0 0}$ & $\mathbf{0 . 9 5 2 0}$ \\
\cline { 2 - 5 } & $\mathrm{Y}$ & 0.1180 & $\mathbf{0 . 2 2 2 0}$ & 0.1540 \\
\hline RF & $\mathrm{N}$ & 0.9140 & 0.8310 & 0.8710 \\
\hline
\end{tabular}

Results of CM1 datasets are given in Table 3. The table reflects that, in Precision, NB performed better in both the classes ( $\mathrm{Y}$ and $\mathrm{N}$ ). In Recall, NB and DT both performed better in $\mathrm{Y}$ class whereas RBF, SVM and PART showed better performance in $\mathrm{N}$ class, and finally in F-measure, NB showed better performance in $\mathrm{Y}$ class

\begin{tabular}{|c|c|c|c|c|}
\hline Classifier & Class & Precision & Recall & F-Measure \\
\hline \multirow[t]{2}{*}{ NB } & $\mathrm{Y}$ & 0.5370 & 0.2260 & 0.3180 \\
\hline & $\mathrm{N}$ & 0.8230 & 0.9490 & 0.8820 \\
\hline \multirow[t]{2}{*}{ MLP } & $\mathrm{Y}$ & 0.7650 & 0.0810 & 0.1460 \\
\hline & $\mathrm{N}$ & 0.8040 & 0.9930 & 0.8890 \\
\hline \multirow[t]{2}{*}{ RBF } & $\mathrm{Y}$ & 0.6940 & 0.1040 & 0.1810 \\
\hline & $\mathrm{N}$ & 0.8070 & 0.9880 & 0.8890 \\
\hline \multirow[t]{2}{*}{ SVM } & $\mathrm{Y}$ & $?$ & 0.0000 & $?$ \\
\hline & $\mathrm{N}$ & 0.7920 & 1.0000 & 0.8840 \\
\hline \multirow[t]{2}{*}{ kNN } & $\mathrm{Y}$ & 0.3630 & 0.3340 & 0.3480 \\
\hline & $\mathrm{N}$ & 0.8290 & 0.8460 & 0.8370 \\
\hline \multirow[t]{2}{*}{ kStar } & $\mathrm{Y}$ & 0.4030 & 0.3170 & 0.3550 \\
\hline & $\mathrm{N}$ & 0.8300 & 0.8760 & 0.8530 \\
\hline \multirow[t]{2}{*}{ OneR } & $\mathrm{Y}$ & 0.3780 & 0.1510 & 0.2160 \\
\hline & $\mathrm{N}$ & 0.8070 & 0.9350 & 0.8660 \\
\hline \multirow[t]{2}{*}{ PART } & $\mathrm{Y}$ & 0.8180 & 0.0190 & 0.0370 \\
\hline & $\mathrm{N}$ & 0.7950 & 0.9990 & 0.8850 \\
\hline \multirow[t]{2}{*}{ DT } & $\mathrm{Y}$ & 0.4960 & 0.2680 & 0.3480 \\
\hline & $\mathrm{N}$ & 0.8280 & 0.9290 & 0.8760 \\
\hline \multirow[t]{2}{*}{ RF } & $\mathrm{Y}$ & 0.5720 & 0.1890 & 0.2840 \\
\hline & $\mathrm{N}$ & 0.8190 & 0.9630 & 0.8850 \\
\hline \multirow[t]{2}{*}{ Boost-RF } & $\mathrm{Y}$ & 0.6010 & 0.1970 & 0.2970 \\
\hline & $\mathrm{N}$ & 0.8210 & 0.9660 & 0.8870 \\
\hline \multirow[t]{2}{*}{ Bag-RF } & $\mathrm{Y}$ & 0.6190 & 0.1780 & 0.2770 \\
\hline & $\mathrm{N}$ & 0.8180 & 0.9710 & 0.8880 \\
\hline \multirow{2}{*}{$\begin{array}{c}\text { Boost-RF- } \\
\text { FS }\end{array}$} & $\mathrm{Y}$ & 0.6010 & 0.1970 & 0.2970 \\
\hline & $\mathrm{N}$ & 0.8210 & 0.9660 & 0.8870 \\
\hline \multirow{2}{*}{$\begin{array}{l}\text { Bag-RF- } \\
\text { FS } \\
\end{array}$} & $\mathrm{Y}$ & 0.6190 & 0.1780 & 0.2770 \\
\hline & $\mathrm{N}$ & 0.8180 & 0.9710 & 0.8880 \\
\hline
\end{tabular}
whereas RBF, SVM and PART performed better in $\mathrm{N}$ class.

Table 4. JM1 Data Results

Results of JM1 datasets are reflected in Table 4. In precision, PART performed better in $\mathrm{Y}$ class whereas kStar performed better in $\mathrm{N}$ class. In Recall, kNN performed better in $\mathrm{Y}$ class and SVM performed better in $\mathrm{N}$ class. In F-measure, kStar outperformed in $\mathrm{Y}$ class whereas MLP and RBF outperformed in N class.

\section{Table 5. KC1 Data Results}

\begin{tabular}{|c|c|c|c|c|}
\hline Classifier & Class & Precision & Recall & F-Measure \\
\hline \multirow{2}{*}{ NB } & $\mathrm{Y}$ & 0.4920 & 0.3370 & 0.4000 \\
\cline { 2 - 5 } & $\mathrm{N}$ & 0.7950 & 0.8810 & 0.8360 \\
\hline \multirow{2}{*}{ MLP } & $\mathrm{Y}$ & 0.6470 & 0.2470 & 0.3580 \\
\cline { 2 - 5 } & $\mathrm{N}$ & 0.7870 & 0.9540 & 0.8630 \\
\hline \multirow{2}{*}{ RBF } & $\mathrm{Y}$ & 0.7780 & 0.2360 & 0.3620 \\
\cline { 2 - 5 } & $\mathrm{N}$ & 0.7890 & 0.9770 & $\mathbf{0 . 8 7 3 0}$ \\
\hline
\end{tabular}




\begin{tabular}{|c|c|c|c|c|}
\hline SVM & $\mathrm{Y}$ & $\mathbf{0 . 8 0 0 0}$ & 0.0450 & 0.0850 \\
\hline & $\mathrm{N}$ & 0.7530 & $\mathbf{0 . 9 9 6 0}$ & 0.8580 \\
\hline \multirow{2}{*}{ kNN } & $\mathrm{Y}$ & 0.3980 & 0.3930 & 0.3950 \\
\cline { 2 - 5 } & $\mathrm{N}$ & 0.7930 & 0.7960 & 0.7950 \\
\hline \multirow{2}{*}{ kStar } & $\mathrm{Y}$ & 0.4490 & $\mathbf{0 . 3 9 3 0}$ & 0.4190 \\
\cline { 2 - 5 } OneR & $\mathrm{N}$ & 0.8010 & 0.8350 & 0.8170 \\
\cline { 2 - 5 } & $\mathrm{Y}$ & 0.4440 & 0.1800 & 0.2560 \\
\hline \multirow{2}{*}{ PART } & $\mathrm{N}$ & 0.7670 & 0.9230 & 0.8380 \\
\cline { 2 - 5 } & $\mathrm{Y}$ & 0.6670 & 0.1570 & 0.2550 \\
\hline \multirow{2}{*}{ DT } & $\mathrm{Y}$ & 0.7710 & 0.9730 & 0.8610 \\
\cline { 2 - 5 } & $\mathrm{N}$ & 0.8330 & 0.3600 & 0.4300 \\
\hline \multirow{2}{*}{ RF } & $\mathrm{Y}$ & 0.6150 & 0.8920 & 0.8450 \\
\cline { 2 - 5 } & $\mathrm{N}$ & $\mathbf{0 . 8 0 8 0}$ & 0.9230 & 0.4540 \\
\hline Boost-RF & $\mathrm{Y}$ & 0.5770 & 0.3370 & 0.8620 \\
\cline { 2 - 5 } & $\mathrm{N}$ & 0.8010 & 0.9150 & 0.8550 \\
\hline Bag-RF & $\mathrm{Y}$ & 0.6440 & 0.3260 & 0.4330 \\
\cline { 2 - 5 } & $\mathrm{N}$ & 0.8030 & 0.9380 & 0.8650 \\
\hline Boost-RF- & $\mathrm{Y}$ & 0.6350 & 0.3710 & $\mathbf{0 . 4 6 8 0}$ \\
\cline { 2 - 5 } FS & $\mathrm{N}$ & 0.8110 & 0.9270 & 0.8650 \\
\hline Bag-RF-FS & $\mathrm{Y}$ & 0.6520 & 0.3370 & 0.4440 \\
\cline { 2 - 5 } & $\mathrm{N}$ & 0.8050 & 0.9380 & 0.8670 \\
\hline
\end{tabular}

Results of $\mathrm{KC} 1$ datasets are given in Table 5. It can be seen that in Precision, SVM outperformed in Y Class whereas RF showed better results in N Class. In Recall, $\mathrm{kNN}$ and kStar performed better in Y class whereas SVM showed better performance in $\mathrm{N}$ class, and finally, in $\mathrm{F}$ measure, Boost-RF-FS performed better in $\mathrm{Y}$ and $\mathrm{RBF}$ outperform in $\mathrm{N}$ class.

Table 6. KC3 Data Results

\begin{tabular}{|c|c|c|c|c|}
\hline Classifier & Class & Precision & Recall & F-Measure \\
\hline \multirow[t]{2}{*}{ NB } & $\mathrm{Y}$ & 0.4440 & 0.4000 & 0.4210 \\
\hline & $\mathrm{N}$ & 0.8780 & 0.8960 & 0.8870 \\
\hline \multirow[t]{2}{*}{ MLP } & $\mathrm{Y}$ & 0.5000 & 0.3000 & 0.3750 \\
\hline & $\mathrm{N}$ & 0.8650 & 0.9380 & 0.9000 \\
\hline \multirow[t]{2}{*}{ RBF } & $\mathrm{Y}$ & 0.0000 & 0.0000 & 0.0000 \\
\hline & $\mathrm{N}$ & 0.8180 & 0.9380 & 0.8740 \\
\hline \multirow[t]{2}{*}{ SVM } & $\mathrm{Y}$ & $?$ & 0.0000 & $?$ \\
\hline & $\mathrm{N}$ & 0.8280 & 1.0000 & 0.9060 \\
\hline \multirow[t]{2}{*}{ kNN } & $\mathrm{Y}$ & 0.3330 & 0.4000 & 0.3640 \\
\hline & $\mathrm{N}$ & 0.8700 & 0.8330 & 0.8510 \\
\hline \multirow[t]{2}{*}{ kStar } & Y & 0.3000 & 0.3000 & 0.3000 \\
\hline & $\mathrm{N}$ & 0.8540 & 0.8540 & 0.8540 \\
\hline \multirow[t]{2}{*}{ OneR } & $\mathrm{Y}$ & 0.5000 & 0.3000 & 0.3750 \\
\hline & $\mathrm{N}$ & 0.8650 & 0.9380 & 0.9000 \\
\hline \multirow[t]{2}{*}{ PART } & $\bar{Y}$ & 0.2500 & 0.1000 & 0.1430 \\
\hline & $\mathrm{N}$ & 0.8330 & 0.9380 & 0.8820 \\
\hline \multirow[t]{2}{*}{ DT } & $\mathrm{Y}$ & 0.3000 & 0.3000 & 0.3000 \\
\hline & $\mathrm{N}$ & 0.8540 & 0.8540 & 0.8540 \\
\hline \multirow[t]{2}{*}{ RF } & $\mathrm{Y}$ & 0.2860 & 0.2000 & 0.2350 \\
\hline & $\mathrm{N}$ & 0.8430 & 0.8960 & 0.8690 \\
\hline \multirow[t]{2}{*}{ Boost-RF } & $\mathrm{Y}$ & 0.3330 & 0.2000 & 0.2500 \\
\hline & $\mathrm{N}$ & 0.8460 & 0.9170 & 0.8800 \\
\hline \multirow[t]{2}{*}{ Bag-RF } & $\mathrm{Y}$ & 0.4000 & 0.2000 & 0.2670 \\
\hline & $\mathrm{N}$ & 0.8490 & 0.9380 & 0.8910 \\
\hline \multirow{2}{*}{$\begin{array}{l}\text { Boost-RF- } \\
\text { FS }\end{array}$} & $\mathrm{Y}$ & 0.4170 & 0.5000 & 0.4550 \\
\hline & $\mathrm{N}$ & 0.8910 & 0.8540 & 0.8720 \\
\hline \multirow{2}{*}{$\begin{array}{l}\text { Bag-RF- } \\
\text { FS }\end{array}$} & $\mathrm{Y}$ & 0.2000 & 0.1000 & 0.1330 \\
\hline & $\mathrm{N}$ & 0.8300 & 0.9170 & 0.8710 \\
\hline
\end{tabular}

Results of KC3 dataset is reflected in Table 6. It is reflected that in Precision, MLP and OneR showed highest performance in $\mathrm{Y}$ class whereas Boost-RF-FS. In Recall, Boost-RF-FS performed better in $\mathrm{Y}$ class and in $\mathrm{N}$ class, SVM outperformed the others. In F-measure,
Boost-RF-FS performed better in Y class whereas SVM performed better in $\mathrm{N}$ class.

Table 7. MC1 Data Results

\begin{tabular}{|c|c|c|c|c|}
\hline Classifier & Class & Precision & Recall & F-Measure \\
\hline \multirow[t]{2}{*}{ NB } & $\mathrm{Y}$ & 0.1560 & 0.3570 & 0.2170 \\
\hline & $\mathrm{N}$ & 0.9840 & 0.9530 & 0.9680 \\
\hline \multirow[t]{2}{*}{ MLP } & $\mathrm{Y}$ & $?$ & 0.0000 & $?$ \\
\hline & $\mathrm{N}$ & 0.9760 & 1.0000 & 0.9880 \\
\hline \multirow[t]{2}{*}{ RBF } & $\mathrm{Y}$ & $?$ & 0.0000 & $?$ \\
\hline & $\mathrm{N}$ & 0.9760 & 1.0000 & 0.9880 \\
\hline \multirow[t]{2}{*}{ SVM } & $\mathrm{Y}$ & $?$ & 0.0000 & $?$ \\
\hline & $\mathrm{N}$ & 0.9760 & 1.0000 & 0.9880 \\
\hline \multirow[t]{2}{*}{ kNN } & $\mathrm{Y}$ & 0.4000 & 0.2860 & 0.3330 \\
\hline & $\mathrm{N}$ & 0.9830 & 0.9900 & 0.9860 \\
\hline \multirow[t]{2}{*}{ kStar } & $\mathrm{Y}$ & 0.2500 & 0.1430 & 0.1820 \\
\hline & $\mathrm{N}$ & 0.9790 & 0.9900 & 0.9840 \\
\hline \multirow[t]{2}{*}{ OneR } & $\mathrm{Y}$ & 0.3330 & 0.1430 & 0.2000 \\
\hline & $\mathrm{N}$ & 0.9790 & 0.9930 & 0.9860 \\
\hline \multirow[t]{2}{*}{ PART } & $\mathrm{Y}$ & 0.4000 & 0.2860 & 0.3330 \\
\hline & $\mathrm{N}$ & 0.9830 & 0.9900 & 0.9860 \\
\hline \multirow[t]{2}{*}{ DT } & $\mathrm{Y}$ & $?$ & 0.0000 & $?$ \\
\hline & $\mathrm{N}$ & 0.9760 & 1.0000 & 0.9880 \\
\hline \multirow[t]{2}{*}{$\mathbf{R F}$} & $\mathrm{Y}$ & 0.0000 & 0.0000 & 0.0000 \\
\hline & $\mathrm{N}$ & 0.9760 & 0.9980 & 0.9870 \\
\hline \multirow[t]{2}{*}{ Boost-RF } & $\mathrm{Y}$ & 0.3330 & 0.0710 & 0.1180 \\
\hline & $\mathrm{N}$ & 0.9780 & 0.9970 & 0.9870 \\
\hline \multirow[t]{2}{*}{ Bag-RF } & $\mathrm{Y}$ & $?$ & 0.0000 & $?$ \\
\hline & $\mathrm{N}$ & 0.9760 & 1.0000 & 0.9880 \\
\hline \multirow{2}{*}{$\begin{array}{c}\text { Boost-RF- } \\
\text { FS }\end{array}$} & $\mathrm{Y}$ & 0.5000 & 0.0710 & 0.1250 \\
\hline & $\mathrm{N}$ & 0.9780 & 0.9980 & $\begin{array}{l}0.9880 \\
\end{array}$ \\
\hline \multirow{2}{*}{$\begin{array}{l}\text { Bag-RF- } \\
\text { FS } \\
\end{array}$} & $\mathrm{Y}$ & $?$ & 0.0000 & $?$ \\
\hline & $\mathrm{N}$ & 0.9760 & 1.0000 & 0.9880 \\
\hline
\end{tabular}

Results of MC1 dataset are reflected in Table 7. In Precision, Boost-RF-FS showed better performance in $\mathrm{Y}$ class whereas NB performed better in N class. In Recall, NB performed better in Y class whereas MLP, RBF, SVM, DT, Bag-RF and Bag-RF-FS performed better in $\mathrm{N}$ class. In F-Measure, $\mathrm{kNN}$ and PART performed better in $\mathrm{Y}$ class whereas MLP, RBF, SVM, DT, Bag-RF, Boost-RF-FS, and Bag-RF-FS performed better in $\mathrm{N}$ class.

Table 8. MC2 Data Results

\begin{tabular}{|c|c|c|c|c|}
\hline Classifier & Class & Precision & Recall & F-Measure \\
\hline \multirow{2}{*}{ NB } & $\mathrm{Y}$ & $\mathbf{0 . 8 3 3 0}$ & 0.3850 & 0.5260 \\
\cline { 2 - 5 } & $\mathrm{N}$ & 0.7420 & $\mathbf{0 . 9 5 8 0}$ & 0.8360 \\
\hline \multirow{3}{*}{ RLPF } & $\mathrm{Y}$ & 0.5000 & 0.5380 & 0.5190 \\
\cline { 2 - 5 } & $\mathrm{N}$ & 0.7390 & 0.7080 & 0.7230 \\
\cline { 2 - 5 } & $\mathrm{Y}$ & 0.8000 & 0.3080 & 0.4400 \\
\hline \multirow{3}{*}{ SVM } & $\mathrm{N}$ & 0.7190 & $\mathbf{0 . 9 5 8 0}$ & 0.8210 \\
\cline { 2 - 5 } & $\mathrm{N}$ & 0.4000 & 0.1540 & 0.2220 \\
\hline \multirow{3}{*}{ kNN } & $\mathrm{Y}$ & 0.6560 & 0.8750 & 0.7500 \\
\cline { 2 - 5 } & $\mathrm{N}$ & 0.7500 & 0.8750 & 0.8080 \\
\hline \multirow{2}{*}{ OneR } & $\mathrm{Y}$ & 0.4000 & 0.3080 & 0.3480 \\
\cline { 2 - 5 } & $\mathrm{N}$ & 0.6670 & 0.7500 & 0.7060 \\
\cline { 2 - 5 } & $\mathrm{Y}$ & 0.5000 & 0.2310 & 0.3160 \\
\hline \multirow{2}{*}{ PART } & $\mathrm{N}$ & 0.6770 & 0.8750 & 0.7640 \\
\cline { 2 - 5 } & $\mathrm{Y}$ & 0.7270 & $\mathbf{0 . 6 1 5 0}$ & $\mathbf{0 . 6 6 7 0}$ \\
\hline \multirow{2}{*}{ DT } & $\mathrm{Y}$ & $\mathbf{0 . 8 0 8 0}$ & 0.8750 & $\mathbf{0 . 8 4 0 0}$ \\
\cline { 2 - 5 } & $\mathrm{N}$ & 0.5000 & 0.3850 & 0.4350 \\
\hline \multirow{2}{*}{ RF } & $\mathrm{Y}$ & 0.5000 & 0.4620 & 0.4800 \\
\cline { 2 - 5 } & $\mathrm{N}$ & 0.7200 & 0.7500 & 0.7350 \\
\hline
\end{tabular}




\begin{tabular}{|c|c|c|c|c|}
\hline Boost-RF & $\mathrm{Y}$ & 0.4550 & 0.3850 & 0.4170 \\
\hline & $\mathrm{N}$ & 0.6920 & 0.7500 & 0.7200 \\
\hline \multirow{2}{*}{ Bag-RF } & $\mathrm{Y}$ & 0.5000 & 0.4620 & 0.4800 \\
\cline { 2 - 5 } & $\mathrm{N}$ & 0.7200 & 0.7500 & 0.7350 \\
\hline \multirow{2}{*}{ Boost-RF- } & $\mathrm{Y}$ & 0.5000 & 0.4620 & 0.4800 \\
\cline { 2 - 5 } FS & $\mathrm{N}$ & 0.7200 & 0.7500 & 0.7350 \\
\hline \multirow{2}{*}{ Bag-RF-FS } & $\mathrm{Y}$ & 0.5380 & 0.5380 & 0.5380 \\
\cline { 2 - 5 } & $\mathrm{N}$ & 0.7500 & 0.7500 & 0.7500 \\
\hline
\end{tabular}

Table 8 reflects the results of MC2 dataset. It can be observed that in precision, NB performed better in $\mathrm{Y}$ class whereas PART performed better in $\mathrm{N}$ class. In Recall, PART performed better in $\mathrm{Y}$ class and NB and $\mathrm{RBF}$ performed better in $\mathrm{N}$ class. and finally, in $\mathrm{F}$ Measure, PART showed highest results in both classes.

Table 9. MW1 Data Results

\begin{tabular}{|c|c|c|c|c|}
\hline Classifier & Class & Precision & Recall & F-Measure \\
\hline \multirow[t]{2}{*}{ NB } & $\mathrm{Y}$ & 0.3330 & 0.6250 & 0.4350 \\
\hline & $\mathrm{N}$ & 0.9500 & 0.8510 & 0.8980 \\
\hline \multirow[t]{2}{*}{ MLP } & $\mathrm{Y}$ & 0.5450 & 0.7500 & 0.6320 \\
\hline & $\mathrm{N}$ & 0.9690 & 0.9250 & 0.9470 \\
\hline \multirow[t]{2}{*}{ RBF } & $\mathrm{Y}$ & $?$ & 0.0000 & $?$ \\
\hline & $\mathrm{N}$ & 0.8930 & 1.0000 & 0.9440 \\
\hline \multirow[t]{2}{*}{ SVM } & $\mathrm{Y}$ & $?$ & 0.0000 & $?$ \\
\hline & $\mathrm{N}$ & 0.8930 & 1.0000 & 0.9440 \\
\hline \multirow[t]{2}{*}{ kNN } & $\mathrm{Y}$ & 0.4000 & 0.5000 & 0.4440 \\
\hline & $\mathrm{N}$ & 0.9380 & 0.9100 & 0.9240 \\
\hline \multirow[t]{2}{*}{ kStar } & $\mathrm{Y}$ & 0.1430 & 0.1250 & 0.1330 \\
\hline & $\mathrm{N}$ & 0.8970 & 0.9100 & 0.9040 \\
\hline \multirow[t]{2}{*}{ OneR } & $\mathrm{Y}$ & 0.5000 & 0.1250 & 0.2000 \\
\hline & $\mathrm{N}$ & 0.9040 & 0.9850 & 0.9430 \\
\hline \multirow[t]{2}{*}{ PART } & $\mathrm{Y}$ & 0.2500 & 0.1250 & 0.1670 \\
\hline & $\mathrm{N}$ & 0.9010 & 0.9550 & 0.9280 \\
\hline \multirow[t]{2}{*}{ DT } & $\mathrm{Y}$ & 0.2500 & 0.1250 & 0.1670 \\
\hline & $\mathrm{N}$ & 0.9010 & 0.9550 & 0.9280 \\
\hline \multirow[t]{2}{*}{ RF } & $\mathrm{Y}$ & 0.3330 & 0.1250 & 0.1820 \\
\hline & $\mathrm{N}$ & 0.9030 & 0.9700 & 0.9350 \\
\hline \multirow[t]{2}{*}{ Boost-RF } & $\mathrm{Y}$ & 0.5000 & 0.2500 & 0.3330 \\
\hline & $\mathrm{N}$ & 0.9150 & 0.9700 & 0.9420 \\
\hline \multirow[t]{2}{*}{ Bag-RF } & $\mathrm{Y}$ & 0.5000 & 0.1250 & 0.2000 \\
\hline & $\mathrm{N}$ & 0.9040 & 0.9850 & 0.9430 \\
\hline \multirow{2}{*}{$\begin{array}{l}\text { Boost-RF- } \\
\text { FS }\end{array}$} & $\mathrm{Y}$ & 0.5000 & 0.2500 & 0.3330 \\
\hline & $\mathrm{N}$ & 0.9150 & 0.9700 & 0.9420 \\
\hline \multirow{2}{*}{$\begin{array}{l}\text { Bag-RF- } \\
\text { FS }\end{array}$} & $\mathrm{Y}$ & 0.5000 & 0.1250 & 0.2000 \\
\hline & $\mathrm{N}$ & 0.9040 & 0.9850 & 0.9430 \\
\hline
\end{tabular}

Table 9 reflects the result of MW1 dataset. It can be seen that in Precision, MLP performed better in both the classes. In Recall, MLP performed better in Y class whereas RBF and SVM performed better in in N class. In F-measure, MLP performed better in both the classes.

Table 10. PC1 Data Results

\begin{tabular}{|c|c|c|c|c|}
\hline Classifier & Class & Precision & Recall & F-Measure \\
\hline \multirow{2}{*}{ NB } & $\mathrm{Y}$ & 0.2800 & $\mathbf{0 . 7 0 0 0}$ & 0.4000 \\
\cline { 2 - 5 } & $\mathrm{N}$ & 0.9830 & 0.9070 & 0.9440 \\
\hline \multirow{2}{*}{ MLP } & $\mathrm{Y}$ & $\mathbf{1 . 0 0 0 0}$ & 0.3000 & 0.4620 \\
\cline { 2 - 5 } & $\mathrm{N}$ & 0.9650 & $\mathbf{1 . 0 0 0 0}$ & $\mathbf{0 . 9 8 2 0}$ \\
\hline \multirow{2}{*}{ RBF } & $\mathrm{Y}$ & 0.3330 & 0.1000 & 0.1540 \\
\cline { 2 - 5 } & $\mathrm{N}$ & 0.9550 & 0.9900 & 0.9720 \\
\hline \multirow{2}{*}{ kNM } & $\mathrm{Y}$ & $?$ & 0.0000 & $?$ \\
\cline { 2 - 5 } & $\mathrm{N}$ & 0.9510 & $\mathbf{1 . 0 0 0 0}$ & 0.9750 \\
\hline kStar & $\mathrm{Y}$ & 0.2730 & 0.3000 & 0.2860 \\
\cline { 2 - 5 } & $\mathrm{N}$ & 0.9640 & 0.9590 & 0.9610 \\
\hline
\end{tabular}

\begin{tabular}{|c|c|c|c|c|}
\hline & $\mathrm{N}$ & 0.9610 & 0.8920 & 0.9250 \\
\hline \multirow{2}{*}{ OneR } & $\mathrm{Y}$ & 0.3330 & 0.1000 & 0.1540 \\
\cline { 2 - 5 } & $\mathrm{N}$ & 0.9550 & 0.9900 & 0.9720 \\
\hline \multirow{2}{*}{ PART } & $\mathrm{Y}$ & 0.3750 & 0.6000 & 0.4620 \\
\cline { 2 - 5 } & $\mathrm{N}$ & 0.9790 & 0.9480 & 0.9630 \\
\hline \multirow{2}{*}{ DT } & $\mathrm{Y}$ & 0.3890 & $\mathbf{0 . 7 0 0 0}$ & $\mathbf{0 . 5 0 0 0}$ \\
\cline { 2 - 5 } & $\mathrm{N}$ & $\mathbf{0 . 9 8 4 0}$ & 0.9430 & 0.9630 \\
\hline \multirow{3}{*}{ Boost-RF } & $\mathrm{Y}$ & 0.7500 & 0.3000 & 0.4290 \\
\cline { 2 - 5 } & $\mathrm{N}$ & 0.9650 & 0.9950 & 0.9800 \\
\cline { 2 - 5 } Bag-RF & $\mathrm{Y}$ & 0.6000 & 0.3000 & 0.4000 \\
\cline { 2 - 5 } & $\mathrm{N}$ & 0.9650 & 0.9900 & 0.9770 \\
\hline \multirow{2}{*}{ Boost-RF- } & $\mathrm{Y}$ & $\mathbf{1 . 0 0 0 0}$ & 0.2000 & 0.3330 \\
\cline { 2 - 5 } FS & $\mathrm{Y}$ & 0.9600 & $\mathbf{1 . 0 0 0 0}$ & 0.9800 \\
\hline Bag-RF- & $\mathrm{N}$ & 0.6000 & 0.3000 & 0.4000 \\
\cline { 2 - 5 } FS & $\mathrm{Y}$ & $\mathbf{1 . 0 0 0 0}$ & 0.2000 & 0.9770 \\
\hline & & 0.9600 & $\mathbf{1 . 0 0 0 0}$ & 0.9800 \\
\hline
\end{tabular}

Results of PC1 datasets are shown in Table 10. It can be seen that in Precision, MLP, Bag-RF, Boost-RF-FS, and Bag-RF-FS performed better in Y class whereas DT performed better in $\mathrm{N}$ class. In Recall, $\mathrm{NB}$ and DT performed better in Y class whereas MLP, SVM, Bag-RF, Boost-RF-FS, and Bag-RF-FS both performed better in $\mathrm{N}$ class. In F-measure, DT performed better in $\mathrm{Y}$ class whereas MLP performed better in N class.

Table 11. PC2 Data Results

\begin{tabular}{|c|c|c|c|c|}
\hline Classifier & Class & Precision & Recall & F-Measure \\
\hline \multirow[t]{2}{*}{ NB } & $\mathrm{Y}$ & 0.0000 & 0.0000 & 0.0000 \\
\hline & $\mathrm{N}$ & 0.9760 & 0.9670 & 0.9720 \\
\hline \multirow[t]{2}{*}{ MLP } & $\mathrm{Y}$ & 0.0000 & 0.0000 & 0.0000 \\
\hline & $\mathrm{N}$ & 0.9770 & 0.9910 & 0.9840 \\
\hline \multirow[t]{2}{*}{ RBF } & $\mathrm{Y}$ & $?$ & 0.0000 & $?$ \\
\hline & $\mathrm{N}$ & 0.9770 & 1.0000 & 0.9880 \\
\hline \multirow[t]{2}{*}{ SVM } & $\mathrm{Y}$ & $?$ & 0.0000 & $?$ \\
\hline & $\mathrm{N}$ & 0.9770 & 1.0000 & 0.9880 \\
\hline \multirow[t]{2}{*}{ kNN } & $\mathrm{Y}$ & 0.0000 & 0.0000 & 0.0000 \\
\hline & $\mathrm{N}$ & 0.9770 & 0.9910 & 0.9840 \\
\hline \multirow[t]{2}{*}{ kStar } & $\mathrm{Y}$ & 0.1430 & 0.2000 & 0.1670 \\
\hline & $\mathrm{N}$ & 0.9810 & 0.9720 & 0.9760 \\
\hline \multirow[t]{2}{*}{ OneR } & $\mathrm{Y}$ & 0.0000 & 0.0000 & 0.0000 \\
\hline & $\mathrm{N}$ & 0.9770 & 0.9950 & 0.9860 \\
\hline \multirow[t]{2}{*}{ PART } & $\mathrm{Y}$ & 0.0000 & 0.0000 & 0.0000 \\
\hline & $\mathrm{N}$ & 0.9770 & 0.9910 & 0.9840 \\
\hline \multirow[t]{2}{*}{ DT } & $\mathrm{Y}$ & $?$ & 0.0000 & $?$ \\
\hline & $\mathrm{N}$ & 0.9770 & 1.0000 & 0.9880 \\
\hline \multirow[t]{2}{*}{$\mathbf{R F}$} & $\mathrm{Y}$ & $?$ & 0.0000 & $?$ \\
\hline & $\mathrm{N}$ & 0.9770 & 1.0000 & 0.9880 \\
\hline \multirow[t]{2}{*}{ Boost-RF } & $\mathrm{Y}$ & $?$ & 0.0000 & $?$ \\
\hline & $\mathrm{N}$ & 0.9770 & 1.0000 & 0.9880 \\
\hline \multirow[t]{2}{*}{ Bag-RF } & $\mathrm{Y}$ & $?$ & 0.0000 & $?$ \\
\hline & $\mathrm{N}$ & 0.9770 & 1.0000 & 0.9880 \\
\hline \multirow{2}{*}{$\begin{array}{c}\text { Boost-RF- } \\
\text { FS }\end{array}$} & $\mathrm{Y}$ & 0.0000 & 0.0000 & 0.0000 \\
\hline & $\mathrm{N}$ & 0.9770 & 0.9950 & 0.9860 \\
\hline \multirow{2}{*}{$\begin{array}{c}\text { Bag-RF- } \\
\text { FS }\end{array}$} & $\mathrm{Y}$ & $?$ & 0.0000 & $?$ \\
\hline & $\mathrm{N}$ & 0.9770 & 1.0000 & 0.9880 \\
\hline
\end{tabular}

Results of PC2 datasets are shown in Table 11. According to results in Precision, kStar performed well in both the classes. In Recall, kStar performed well in $\mathrm{Y}$ class whereas RBF, SVM, DT, RF, Boost-RF, Bag-RF, and Bag-RF-FS performed well in N class. In F-measure, kStar performed well in Y class however RBF, SVM, DT, RF, Boost-RF, Bag-RF, and Bag-RF-FS performed well in $\mathrm{N}$ class. 
Table 12. PC3 Data Results

\begin{tabular}{|c|c|c|c|c|}
\hline Classifier & Class & Precision & Recall & F-Measure \\
\hline \multirow[t]{2}{*}{ NB } & Y & 0.1500 & 0.9070 & 0.2570 \\
\hline & $\mathrm{N}$ & 0.9290 & 0.1900 & 0.3160 \\
\hline \multirow[t]{2}{*}{ MLP } & $\mathrm{Y}$ & 0.3460 & 0.2090 & 0.2610 \\
\hline & $\mathrm{N}$ & 0.8830 & 0.9380 & 0.9090 \\
\hline \multirow[t]{2}{*}{ RBF } & $\bar{Y}$ & $?$ & 0.0000 & $?$ \\
\hline & $\mathrm{N}$ & 0.8640 & 1.0000 & 0.9270 \\
\hline \multirow[t]{2}{*}{ SVM } & $\mathrm{Y}$ & $?$ & 0.0000 & $?$ \\
\hline & $\mathrm{N}$ & 0.8640 & 1.0000 & 0.9270 \\
\hline \multirow[t]{2}{*}{ kNN } & $\mathrm{Y}$ & 0.4800 & 0.2790 & 0.3530 \\
\hline & $\mathrm{N}$ & 0.8930 & 0.9520 & 0.9220 \\
\hline \multirow[t]{2}{*}{ kStar } & $\mathrm{Y}$ & 0.3130 & 0.2330 & 0.2670 \\
\hline & $\mathrm{N}$ & 0.8840 & 0.9190 & 0.9010 \\
\hline \multirow[t]{2}{*}{ OneR } & $\mathrm{Y}$ & 0.6000 & 0.1400 & 0.2260 \\
\hline & $\mathrm{N}$ & 0.8790 & 0.9850 & 0.9290 \\
\hline \multirow[t]{2}{*}{ PART } & $\mathrm{Y}$ & $?$ & 0.0000 & $?$ \\
\hline & $\mathrm{N}$ & 0.8640 & 1.0000 & 0.9270 \\
\hline \multirow[t]{2}{*}{ DT } & $\mathrm{Y}$ & 0.5000 & 0.2790 & 0.3580 \\
\hline & $\mathrm{N}$ & 0.8940 & 0.9560 & 0.9240 \\
\hline \multirow[t]{2}{*}{$\mathbf{R F}$} & Y & 0.6000 & 0.1400 & 0.2260 \\
\hline & $\mathrm{N}$ & 0.8790 & 0.9850 & 0.9290 \\
\hline \multirow[t]{2}{*}{ Boost-RF } & $\mathrm{Y}$ & 0.4440 & 0.0930 & 0.1540 \\
\hline & $\mathrm{N}$ & 0.8730 & 0.9820 & 0.9240 \\
\hline \multirow[t]{2}{*}{ Bag-RF } & $\mathrm{Y}$ & 0.5710 & 0.0930 & 0.1600 \\
\hline & $\mathrm{N}$ & 0.8740 & 0.9890 & 0.9280 \\
\hline \multirow{2}{*}{$\begin{array}{l}\text { Boost-RF- } \\
\text { FS }\end{array}$} & $\mathrm{Y}$ & 0.6670 & 0.1400 & 0.2310 \\
\hline & $\mathrm{N}$ & 0.8790 & 0.9890 & 0.9310 \\
\hline \multirow{2}{*}{$\begin{array}{l}\text { Bag-RF- } \\
\text { FS }\end{array}$} & $\mathrm{Y}$ & 0.8000 & 0.0930 & 0.1670 \\
\hline & $\mathrm{N}$ & 0.8750 & 0.9960 & 0.9320 \\
\hline
\end{tabular}

Results of PC3 dataset is reflected in Table 12. It can be seen that in Precision, Bag-RF-FS performed better in $\mathrm{Y}$ class however NB performed better in $\mathrm{N}$ class. In Recall, NB performed better in $\mathrm{Y}$ class whereas RBF, SVM and PART performed better in $\mathrm{N}$ class. In $\mathrm{F}$ measure, DT performed better in Y class whereas BagRF-FS performed better in $\mathrm{N}$ class.

Table 13. PC4 Data Results

\begin{tabular}{|c|c|c|c|c|}
\hline Classifier & Class & Precision & Recall & F-Measure \\
\hline \multirow{2}{*}{ NB } & $\mathrm{Y}$ & 0.4860 & 0.3460 & 0.4040 \\
\cline { 2 - 5 } & $\mathrm{N}$ & 0.9010 & 0.9420 & 0.9210 \\
\hline \multirow{3}{*}{ MLP } & $\mathrm{Y}$ & 0.6760 & 0.4810 & 0.5620 \\
\cline { 2 - 5 } & $\mathrm{N}$ & 0.9220 & 0.9640 & 0.9420 \\
\hline \multirow{3}{*}{ SVM } & $\mathrm{Y}$ & 0.6670 & 0.1540 & 0.2500 \\
\cline { 2 - 5 } & $\mathrm{N}$ & 0.8810 & 0.9880 & 0.9310 \\
\hline \multirow{3}{*}{ kNN } & $\mathrm{Y}$ & 0.8180 & 0.1730 & 0.2860 \\
\cline { 2 - 5 } & $\mathrm{N}$ & 0.8840 & $\mathbf{0 . 9 9 4 0}$ & 0.9360 \\
\cline { 2 - 5 } kStar & $\mathrm{Y}$ & 0.4770 & 0.4040 & 0.4380 \\
\cline { 2 - 5 } & $\mathrm{N}$ & 0.9080 & 0.9300 & 0.9190 \\
\cline { 2 - 5 } OneR & $\mathrm{Y}$ & 0.3330 & 0.3270 & 0.3300 \\
\cline { 2 - 5 } & $\mathrm{Y}$ & 0.8940 & 0.8970 & 0.8950 \\
\hline \multirow{2}{*}{ PART } & $\mathrm{N}$ & 0.8920 & 0.2500 & 0.3610 \\
\cline { 2 - 5 } & $\mathrm{Y}$ & 0.4640 & 0.5000 & 0.9330 \\
\hline \multirow{2}{*}{ DT } & $\mathrm{N}$ & 0.9200 & 0.9090 & 0.9140 \\
\cline { 2 - 5 } & $\mathrm{Y}$ & 0.5150 & $\mathbf{0 . 6 7 3 0}$ & 0.5830 \\
\hline
\end{tabular}

\begin{tabular}{|c|c|c|c|c|}
\hline \multirow{2}{*}{ RF } & $\mathrm{Y}$ & 0.7780 & 0.4040 & 0.5320 \\
\cline { 2 - 5 } & $\mathrm{N}$ & 0.9120 & 0.9820 & 0.9460 \\
\hline \multirow{2}{*}{ Boost-RF } & $\mathrm{Y}$ & 0.7880 & 0.5000 & $\mathbf{0 . 6 1 2 0}$ \\
\cline { 2 - 5 } & $\mathrm{N}$ & 0.9250 & 0.9790 & 0.9510 \\
\hline \multirow{2}{*}{ Bag-RF } & $\mathrm{Y}$ & 0.8570 & 0.3460 & 0.4930 \\
\cline { 2 - 5 } & $\mathrm{N}$ & 0.9060 & 0.9910 & 0.9460 \\
\hline \multirow{2}{*}{ Boost-RF- } & $\mathrm{Y}$ & 0.8330 & 0.4810 & 0.6100 \\
\cline { 2 - 5 } FS & $\mathrm{N}$ & 0.9230 & 0.9850 & $\mathbf{0 . 9 5 3 0}$ \\
\hline Bag-RF-FS & $\mathrm{Y}$ & $\mathbf{0 . 9 0 5 0}$ & 0.3650 & 0.5210 \\
\cline { 2 - 5 } & $\mathrm{N}$ & 0.9080 & $\mathbf{0 . 9 9 4 0}$ & 0.9490 \\
\hline
\end{tabular}

Results of PC4 datasets are shown in Table 13. It can be seen that in Precision, Bag-RF-FS performed better in $\mathrm{Y}$ class whereas DT performed better in $\mathrm{N}$ class. In Recall, DT performed better in Y class whereas SVM and Bag-RF-FS performed better in $\mathrm{N}$ class, and finally, In Fmeasure, Boosting-RF performed better in $\mathrm{Y}$ class whereas Boosting-RF-FS performed better in N class.

Table 14. PC5 Data Results

\begin{tabular}{|c|c|c|c|c|}
\hline Classifier & Class & Precision & Recall & F-Measure \\
\hline \multirow[t]{2}{*}{ NB } & $\mathrm{Y}$ & 0.6760 & 0.1680 & 0.2690 \\
\hline & $\mathrm{N}$ & 0.7590 & 0.9700 & 0.8520 \\
\hline \multirow[t]{2}{*}{ MLP } & $\mathrm{Y}$ & 0.5600 & 0.2040 & 0.2990 \\
\hline & $\mathrm{N}$ & 0.7620 & 0.9410 & 0.8420 \\
\hline \multirow[t]{2}{*}{ RBF } & $\bar{Y}$ & 0.7600 & 0.1390 & 0.2350 \\
\hline & $\mathrm{N}$ & 0.7560 & 0.9840 & 0.8550 \\
\hline \multirow[t]{2}{*}{ SVM } & $\mathrm{Y}$ & 0.8750 & 0.0510 & 0.0970 \\
\hline & $\mathrm{N}$ & 0.7400 & 0.9970 & 0.8500 \\
\hline \multirow[t]{2}{*}{ kNN } & $\mathrm{Y}$ & 0.5000 & 0.4960 & 0.4980 \\
\hline & $\mathrm{N}$ & 0.8150 & 0.8170 & 0.8160 \\
\hline \multirow[t]{2}{*}{ kStar } & $\mathrm{Y}$ & 0.4390 & 0.4230 & 0.4310 \\
\hline & $\mathrm{N}$ & 0.7900 & 0.8010 & 0.7950 \\
\hline \multirow[t]{2}{*}{ OneR } & $\mathrm{Y}$ & 0.4550 & 0.3360 & 0.3870 \\
\hline & $\mathrm{N}$ & 0.7760 & 0.8520 & 0.8120 \\
\hline \multirow[t]{2}{*}{ PART } & $\mathrm{Y}$ & 0.6460 & 0.2260 & 0.3350 \\
\hline & $\mathrm{N}$ & 0.7700 & 0.9540 & 0.8520 \\
\hline \multirow[t]{2}{*}{ DT } & $\mathrm{Y}$ & 0.5370 & 0.5260 & 0.5310 \\
\hline & $\mathrm{N}$ & 0.8260 & 0.8330 & 0.8300 \\
\hline \multirow[t]{2}{*}{ RF } & $\mathrm{Y}$ & 0.5880 & 0.3650 & 0.4500 \\
\hline & $\mathrm{N}$ & 0.7940 & 0.9060 & 0.8460 \\
\hline \multirow[t]{2}{*}{ Boost-RF } & $\mathrm{Y}$ & 0.5880 & 0.3430 & 0.4330 \\
\hline & $\mathrm{N}$ & 0.7900 & 0.9110 & 0.8460 \\
\hline \multirow[t]{2}{*}{ Bag-RF } & $\mathrm{Y}$ & 0.6430 & 0.3280 & 0.4350 \\
\hline & $\mathrm{N}$ & 0.7900 & 0.9330 & 0.8550 \\
\hline \multirow{2}{*}{$\begin{array}{c}\text { Boost-RF- } \\
\text { FS }\end{array}$} & $\mathrm{Y}$ & 0.5880 & 0.3430 & 0.4330 \\
\hline & $\mathrm{N}$ & 0.7900 & 0.9110 & 0.8460 \\
\hline \multirow[t]{2}{*}{ Bag-RF-FS } & $\mathrm{Y}$ & 0.6430 & 0.3280 & 0.4350 \\
\hline & $\mathrm{N}$ & 0.7900 & 0.9330 & 0.8550 \\
\hline
\end{tabular}

Results of PC5 dataset are presented in Table 14. It can be seen that in Precision, SVM performed better in Y class whereas DT performed better in N class. In Recall, DT performed better in Y class whereas SVM performed better in $\mathrm{N}$ Class, and finally, in F-Measure, DT performed better in $\mathrm{Y}$ class whereas $\mathrm{RBF}$, Bagging-RF and Bagging-RF-FS outperform in $\mathrm{N}$ class. 
Table 15. Accuracy Results

\begin{tabular}{|c|c|c|c|c|c|c|c|c|c|c|c|c|c|c|}
\hline Dataset & NB & MLP & RBF & SVM & kNN & kStar & OneR & PART & DT & RF & $\begin{array}{l}\text { Boost- } \\
\text { RF }\end{array}$ & $\begin{array}{l}\text { Bag- } \\
\text { RF }\end{array}$ & $\begin{array}{l}\text { Boost- } \\
\text { RF-FS }\end{array}$ & $\begin{array}{l}\text { Bag- } \\
\text { RF-FS }\end{array}$ \\
\hline CM1 & 82.6531 & 86.7347 & 90.8163 & 90.8163 & 77.5510 & 77.5510 & 85.7143 & 90.8163 & 77.5510 & 89.7959 & 89.7959 & 89.7959 & 89.7959 & 89.7959 \\
\hline JM1 & 79.8359 & 80.3541 & 80.3972 & 79.1883 & 73.9637 & 75.9931 & 77.1589 & 79.4905 & 79.1019 & 80.1813 & 80.5699 & 80.6131 & 80.5699 & 80.6131 \\
\hline KC1 & 74.2120 & 77.3639 & 78.7966 & 75.3582 & 69.3410 & 72.2063 & 73.3524 & 76.5043 & 75.6447 & 77.9370 & 76.7900 & 78.2235 & 78.5100 & 78.5100 \\
\hline KC3 & 81.0345 & 82.7586 & 77.5862 & 82.7586 & 75.8621 & 75.8621 & 82.7586 & 79.3103 & 75.8621 & 77.5862 & 79.3103 & 81.0345 & 79.3103 & 77.5862 \\
\hline MC2 & 75.6757 & 64.8649 & 72.9730 & 62.1622 & 72.9730 & 59.4595 & 64.8649 & 78.3784 & 64.8649 & 64.8649 & 62.1622 & 64.8649 & 64.8649 & 67.5676 \\
\hline MW1 & 82.6667 & 90.6667 & 89.3333 & 89.3333 & 86.6667 & 82.6667 & 89.3333 & 86.6667 & 86.6667 & 88.0000 & 89.3333 & 89.3333 & 89.3333 & 89.3333 \\
\hline PC1 & 89.7059 & 96.5686 & 94.6078 & 95.0980 & 92.6471 & 86.2745 & 94.6078 & 93.1373 & 93.1373 & 96.0784 & 95.5882 & 96.0784 & 96.0784 & 96.0784 \\
\hline PC2 & 94.4700 & 96.7742 & 97.6959 & 97.6959 & 96.7742 & 95.3917 & 97.2350 & 96.7742 & 97.6959 & 97.6959 & 97.6959 & 97.6959 & 97.2350 & 97.6959 \\
\hline PC3 & 28.7975 & 83.8608 & 86.3924 & 86.3924 & 86.0759 & 82.5949 & 87.0253 & 86.3924 & 86.3924 & 87.0253 & 86.0759 & 86.7089 & 87.3418 & 87.3418 \\
\hline PC4 & 86.0892 & 89.7638 & 87.4016 & 88.189 & 85.8268 & 81.8898 & 87.9265 & 85.3018 & 86.8766 & 90.2887 & 91.3386 & 90.2887 & 91.6010 & 90.8136 \\
\hline PC5 & 75.3937 & 74.2126 & 75.5906 & 74.2126 & 73.0315 & 69.8819 & 71.2598 & 75.7874 & 75.0000 & 75.9843 & 75.7874 & 76.9685 & 75.7874 & 76.9685 \\
\hline
\end{tabular}

Table 16. ROC Area Results

\begin{tabular}{|c|c|c|c|c|c|c|c|c|c|c|c|c|c|c|}
\hline Dataset & NB & MLP & RBF & SVM & kNN & kStar & OneR & PART & DT & RF & $\begin{array}{c}\text { Boost- } \\
\text { RF } \\
\end{array}$ & $\begin{array}{c}\text { Bag- } \\
\text { RF }\end{array}$ & $\begin{array}{l}\text { Boost- } \\
\text { RF-FS }\end{array}$ & $\begin{array}{c}\text { Bag- } \\
\text { RF-FS }\end{array}$ \\
\hline CM1 & 0.7030 & 0.6340 & 0.7020 & 0.5000 & 0.4770 & 0.5380 & 0.4720 & 0.6100 & 0.3780 & 0.7610 & 0.7650 & 0.7370 & 0.6600 & 0.6830 \\
\hline JM1 & 0.6630 & 0.7020 & 0.7130 & 0.5000 & 0.5910 & 0.5720 & 0.5430 & 0.7140 & 0.6710 & 0.7380 & 0.7360 & 0.7460 & 0.7360 & 0.7460 \\
\hline $\mathrm{KC1}$ & 0.6940 & 0.7360 & 0.7130 & 0.5210 & 0.5950 & 0.6510 & 0.5510 & 0.6360 & 0.6060 & 0.7510 & 0.7510 & 0.7570 & 0.7510 & 0.7500 \\
\hline KC3 & 0.7690 & 0.7330 & 0.7350 & 0.5000 & 0.617 & 0.5280 & 0.6190 & 0.7880 & 0.5700 & 0.8070 & 0.7850 & 0.8150 & 0.8340 & 0.8670 \\
\hline MC2 & 0.7950 & 0.7530 & 0.7660 & 0.5140 & 0.6680 & 0.5100 & 0.5530 & 0.7240 & 0.6150 & 0.6460 & 0.6650 & 0.6700 & 0.6460 & 0.6570 \\
\hline MW1 & 0.7910 & 0.8430 & 0.8080 & 0.5000 & 0.7050 & 0.5430 & 0.5550 & 0.3140 & 0.3140 & 0.7660 & 0.7260 & 0.7420 & 0.7260 & 0.7610 \\
\hline PC1 & 0.8790 & 0.7790 & 0.8750 & 0.5000 & 0.6290 & 0.6730 & 0.5450 & 0.8890 & 0.7180 & 0.8580 & 0.8960 & 0.9210 & 0.9240 & 0.9100 \\
\hline PC2 & 0.7510 & 0.7460 & 0.7240 & 0.5000 & 0.4950 & 0.7910 & 0.4980 & 0.6230 & 0.5790 & 0.7310 & 0.6560 & 0.7740 & 0.4890 & 0.5630 \\
\hline PC4 & 0.8070 & 0.8980 & 0.8620 & 0.5830 & 0.6670 & 0.7340 & 0.6140 & 0.7760 & 0.8340 & 0.9450 & 0.9450 & 0.9530 & 0.9520 & 0.9550 \\
\hline PC5 & 0.7250 & 0.7510 & 0.7320 & 0.5240 & 0.6570 & 0.6290 & 0.5940 & 0.7390 & 0.7030 & 0.8050 & 0.7990 & 0.8050 & 0.7990 & 0.8050 \\
\hline
\end{tabular}

Table 17. MCC Results

\begin{tabular}{|c|c|c|c|c|c|c|c|c|c|c|c|c|c|c|}
\hline Dataset & NB & MLP & RBF & SVM & kNN & kStar & OneR & PART & DT & RF & $\begin{array}{c}\text { Boost- } \\
\text { RF }\end{array}$ & Bag-RF & $\begin{array}{l}\text { Boost- } \\
\text { RF-FS }\end{array}$ & $\begin{array}{c}\text { Bag- } \\
\text { RF-FS }\end{array}$ \\
\hline CM1 & 0.0970 & -0.0660 & $?$ & $?$ & -0.0370 & -0.037 & -0.074 & $?$ & 0.0410 & -0.032 & -0.032 & -0.032 & -0.032 & -0.032 \\
\hline JM1 & 0.2510 & 0.2060 & 0.2150 & $?$ & 0.1860 & 0.2120 & 0.1260 & 0.1040 & 0.2520 & 0.2440 & 0.2620 & 0.2560 & 0.2620 & 0.2560 \\
\hline $\mathrm{KC1}$ & 0.2500 & 0.2960 & 0.3470 & 0.1510 & 0.1900 & 0.2380 & 0.1470 & 0.2390 & 0.2910 & 0.3460 & 0.3090 & 0.3440 & 0.3640 & 0.3550 \\
\hline $\mathrm{KC3}$ & 0.3090 & 0.2950 & -0.1070 & $?$ & 0.2180 & 0.1540 & 0.2950 & 0.0560 & 0.1540 & 0.1110 & 0.1450 & 0.1850 & 0.3300 & 0.0220 \\
\hline MC1 & 0.2080 & $?$ & $?$ & $?$ & 0.3250 & 0.1740 & 0.2060 & 0.3250 & $?$ & -0.006 & 0.1450 & $?$ & 0.1820 & $?$ \\
\hline MC2 & 0.4440 & 0.2430 & 0.3710 & 0.0400 & 0.3740 & 0.0620 & 0.1370 & 0.5120 & 0.1890 & 0.2160 & 0.1410 & 0.2160 & 0.2160 & 0.2880 \\
\hline MW1 & 0.3670 & 0.5890 & $?$ & $?$ & 0.3730 & 0.0380 & 0.2110 & 0.1100 & 0.1100 & 0.1500 & 0.3020 & 0.2110 & 0.3020 & 0.2110 \\
\hline PC1 & 0.4000 & 0.5380 & 0.1610 & $?$ & 0.2470 & 0.1280 & 0.1610 & 0.4400 & 0.4900 & 0.4590 & 0.4050 & 0.4380 & 0.4380 & 0.4380 \\
\hline PC2 & -0.0280 & -0.0150 & $?$ & $?$ & -0.0150 & 0.1460 & -0.010 & $\begin{array}{c}- \\
0.0150 \\
\end{array}$ & $?$ & $?$ & $?$ & $?$ & -0.010 & $?$ \\
\hline PC3 & 0.0880 & 0.1830 & $?$ & $?$ & 0.2940 & 0.1730 & 0.2450 & $?$ & 0.3040 & 0.2450 & 0.1540 & 0.1910 & 0.2650 & 0.2460 \\
\hline PC4 & 0.3340 & 0.5150 & 0.2790 & 0.3420 & 0.3590 & 0.2250 & 0.3520 & 0.3960 & 0.5140 & 0.5160 & 0.5840 & 0.5070 & 0.5930 & 0.5410 \\
\hline PC5 & 0.2450 & 0.2160 & 0.2510 & 0.1730 & 0.3140 & 0.2270 & 0.2090 & 0.2740 & 0.3610 & 0.3220 & 0.3100 & 0.3360 & 0.3100 & 0.3360 \\
\hline
\end{tabular}


We have considered F-measure for analysis from Table 3 to Table 14 with 'Yes' class. F measure is selected as it provides the average of Precision and Recall and 'Yes' class predicts the probability of defective modules. It has been observed from the results of F-measure that the proposed framework outperformed only in three datasets KC1, KC3 and PC4. In Accuracy (Table 15), the proposed framework performed better in four datasets including JM1, PC3, PC4, and PC5. In remaining datasets either the result is lower or equal to one or more of the other classification techniques. It has also been noted that $\mathrm{NB}, \mathrm{kNN}$, and $\mathrm{kStar}$ could not be able to perform better in any of the dataset. In ROC Area, the higher performance is reflected in the following datasets: CM1, JM1, KC1, KC3, MC1, PC1, and PC4 however the results in remaining datasets shows either lower or equal performance when compared to other classification techniques. It has also been observed that RBF, SVM, kNN, OneR, PART, and DT could not be able to perform better in any of the dataset. In MCC, the proposed framework showed the higher performance in following datasets: JM1, KC1, KC3 and PC4. In remaining datasets the scores are either lower or equal, as compared to other classification techniques. It has also been noted that RBF, SVM, OneR, RF, Bag-RF, and Bag-RF-FS could not be able to perform better in any of the dataset.

As discussed by [10], F- measure and MCC reacts to the issue of class imbalance however it has been observed in this study that our proposed framework could not be able to fully solve that issue either.

\section{CONCLUSION}

This research proposed and implemented a feature selection based ensemble classification framework. The proposed framework consisted of four stages including: 1) Dataset, 2) Feature Selection, 3) Classification, and 4) Results. Two different dimensions are used in the framework, one with feature selection and second without feature selection. Each dimension further used two ensemble techniques with Random Forest classifier: Bagging and Boosting. Performance of proposed framework is evaluated through Precision, Recall, Fmeasure, Accuracy, MCC and ROC. For experiments, 12 Cleaned publically available NASA datasets are used and the results of both the dimensions are compared with the other widely used classification techniques such as: "Naïve Bayes (NB), Multi-Layer Perceptron (MLP). Radial Basis Function (RBF), Support Vector Machine (SVM), K Nearest Neighbor (KNN), kStar (K*), One Rule (OneR), PART, Decision Tree (DT), and Random Forest (RF)". Results showed that the proposed classification framework outperformed other classification techniques in some of the datasets however class imbalance issue could not be resolved, which is the main reason of lower and biased performance of classification techniques. It is suggested for future work that the resampling techniques should be included in proposed framework to resolve the class imbalance issue in datasets as well as to achieve higher performance.

\section{REFERENCES}

[1] S. Huda, S. Alyahya, M. M. Ali, S. Ahmad, J. Abawajy, J. Al-Dossari, and J. Yearwood, "A Framework for Software Defect Prediction and Metric Selection," IEEE Access, vol. 6, pp. 2844-2858, 2018.

[2] E. Erturk and E. Akcapinar, "A comparison of some soft computing methods for software fault prediction," Expert Syst. Appl., vol. 42, no. 4, pp. 1872-1879, 2015.

[3] Y. Ma, G. Luo, X. Zeng, and A. Chen, "Transfer learning for crosscompany software defect prediction," Inf. Softw. Technol., vol. 54, no. 3, Mar. 2012.

[4] M. Shepperd, Q. Song, Z. Sun and C. Mair, "Data Quality: Some Comments on the NASA Software Defect Datasets," IEEE Trans. Softw. Eng., vol. 39, pp. 1208-1215, 2013.

[5] I.H. Witten and E. Frank, Data Mining: Practical Machine Learning Tools and Techniques, second ed. Morgan Kaufmann, 2005.

[6] "NASA Defect Dataset." [Online]. Available: https://github.com/klainfo/NASADefectDataset. [Accessed: 01-July-2019].

[7] B. Ghotra, S. McIntosh, and A. E. Hassan, "Revisiting the impact of classification techniques on the performance of defect prediction models," Proc. - Int. Conf. Softw. Eng., vol. 1, pp. 789-800, 2015.

[8] G. Czibula, Z. Marian, and I. G. Czibula, "Software defect prediction using relational association rule mining," Inf. Sci. (Ny)., vol. 264, pp. 260-278, 2014.

[9] D. Rodriguez, I. Herraiz, R. Harrison, J. Dolado, and J. C. Riquelme, "Preliminary comparison of techniques for dealing with imbalance in software defect prediction," in Proceedings of the 18th International Conference on Evaluation and Assessment in Software Engineering. ACM, p. 43, 2014.

[10] A. Iqbal, S. Aftab, U. Ali, Z. Nawaz, L. Sana, M. Ahmad, and A. Husen "Performance Analysis of Machine Learning Techniques on Software Defect Prediction using NASA Datasets," Int. J. Adv. Comput. Sci. Appl., vol. 10, no. 5, 2019.

[11] M. Ahmad, S. Aftab, I. Ali, and N. Hameed, "Hybrid Tools and Techniques for Sentiment Analysis: A Review," Int. J. Multidiscip. Sci. Eng., vol. 8, no. 3, 2017.

[12] M. Ahmad, S. Aftab, and S. S. Muhammad, "Machine Learning Techniques for Sentiment Analysis: A Review," Int. J. Multidiscip. Sci. Eng., vol. 8, no. 3, p. 27, 2017.

[13] M. Ahmad, S. Aftab, and I. Ali, "Sentiment Analysis of Tweets using SVM," Int. J. Comput. Appl., vol. 177, no. 5, pp. 25-29, 2017.

[14] M. Ahmad and S. Aftab, "Analyzing the Performance of SVM for Polarity Detection with Different Datasets," Int. J. Mod. Educ. Comput. Sci., vol. 9, no. 10, pp. 29-36, 2017.

[15] M. Ahmad, S. Aftab, M. S. Bashir, N. Hameed, I. Ali, and Z. Nawaz, "SVM Optimization for Sentiment Analysis," Int. J. Adv. Comput. Sci. Appl., vol. 9, no. 4, 2018.

[16] M. Ahmad, S. Aftab, M. S. Bashir, and N. Hameed, "Sentiment Analysis using SVM: A Systematic Literature Review," Int. J. Adv. Comput. Sci. Appl., vol. 9, no. 2, 2018.

[17] A. Iqbal and S. Aftab, "A Feed-Forward and Pattern Recognition ANN Model for Network Intrusion Detection,” Int. J. Comput. Netw. Inf. Secur., vol. 11, no. 4, pp. 19-25, 2019. 
[18] A. Iqbal, S. Aftab, I. Ullah, M. A. Saeed, and A. Husen, "A Classification Framework to Detect DoS Attacks," Int. J. Comput. Netw. Inf. Secur., vol. 11, no. 9, pp. 40-47, 2019.

[19] S. Behal, K. Kumar, and M. Sachdeva, "D-FAC: A novel $\phi$-Divergence based distributed DDoS defense system," J. King Saud Univ. - Comput. Inf. Sci., 2018.

[20] S. Aftab, M. Ahmad, N. Hameed, M. S. Bashir, I. Ali, and Z. Nawaz, "Rainfall Prediction in Lahore City using Data Mining Techniques," Int. J. Adv. Comput. Sci. Appl., vol. 9, no. 4, 2018.

[21] S. Aftab, M. Ahmad, N. Hameed, M. S. Bashir, I. Ali, and Z. Nawaz, "Rainfall Prediction using Data Mining Techniques: A Systematic Literature Review," Int. J. Adv. Comput. Sci. Appl., vol. 9, no. 5, 2018.

[22] S. Wang and X. Yao, "Using class imbalance learning for software defect prediction," IEEE Transactions on Reliability, vol. 62, no. 2, pp. 434-443, 2013.

[23] J. C. Riquelme, R. Ruiz, D. Rodr'iguez, and J. Moreno, "Finding defective modules from highly unbalanced datasets," Actas de los Talleres de las Jornadas de Ingenier'1a del Software y Bases de Datos, vol. 2, no. 1, pp. 67-74, 2008

[24] F. Lanubile, A. Lonigro, and G. Visaggio, "Comparing Models for Identifying Fault-Prone Software Components," Proc. Seventh Int'l Conf. Software Eng. and Knowledge Eng., pp. 312-319, June 1995.

[25] K. O. Elish and M. O. Elish, "Predicting defect-prone software modules using support vector machines," J. Syst. Softw., vol. 81, no. 5, pp. 649-660, 2008.

[26] I. Gondra, "Applying machine learning to software faultproneness prediction,” J. Syst. Softw., vol. 81, no. 2, pp. 186-195, 2008.

[27] O. F. Arar and K. Ayan, "Software defect prediction using cost-sensitive ". neural network," Applied Soft Computing, vol. 33, pp. 263-277, 2015.

[28] C. Manjula and L. Florence, "Deep neural network based hybrid approach for software defect prediction using software metrics," Cluster Comput., pp. 1-17, 2018.

[29] R. Moser, W. Pedrycz, and G. Succi, "A Comparative Analysis of the Efficiency of Change Metrics and Static Code Attributes for Defect Prediction", In Robby, editor, ICSE, pp. 181-190. ACM, 2008.

[30] E. Giger, M. D'Ambros, M. Pinzger, and H. C. Gall, "Method-level bug prediction," in ESEM '12, pp. 171$180,2012$.

[31] S. E. S. Taba, F. Khomh, Y. Zou, A. E. Hassan, and M. Nagappan, "Predicting bugs using antipatterns," in Proc. of the 29th Int'l Conference on Software Maintenance, pp. 270-279, 2013.

[32] K. Herzig, S. Just, A. Rau, and A. Zeller, "Predicting defects using change genealogies," in Software Reliability Engineering (ISSRE), 2013 IEEE 24th International Symposium on, pp. 118-127, 2013.

\section{Authors' Profiles}

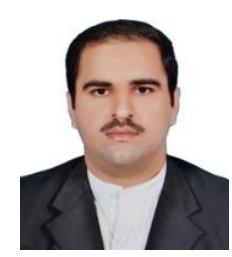

Ahmed Iqbal is student of MS Computer Science with the specialization of Software Engineering in Virtual University of Pakistan. He received the degree of 'Master of Information Technology '(MIT) from Virtual University of Pakistan in 2016. His research interest includes Software
Engineering and Data Mining.

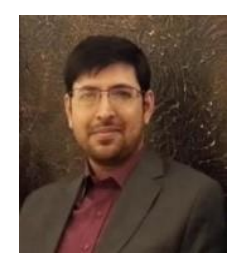

Shabib Aftab received MS Degree in Computer Science from COMSATS Institute of Information Technology Lahore, Pakistan, and M.Sc degree in Information Technology from Punjab University College of Information Technology (PUCIT) Lahore, Pakistan. Currently he is serving as Lecturer Computer Sciences at Virtual University of Pakistan. His research areas include Data Mining and Software Process Improvement.

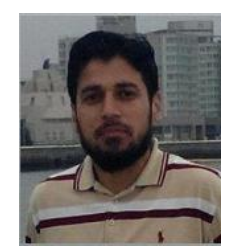

Israr Ullah has completed PhD in Computer Engineering from Jeju National University, South Korea in February 2019. He completed his M.S. in Computer Science from National University of Computer and Emerging Sciences (NUCES), Islamabad, Pakistan in 2009. He is serving as Assistant Professor of Computer Science at Virtual University of Pakistan His research is mainly focused on development of AI based IoT solutions for smart cities. He has experience in the field of Network Simulation and Modeling. His research interests also include Designing and Analysis of Optimization Algorithms using AI techniques.

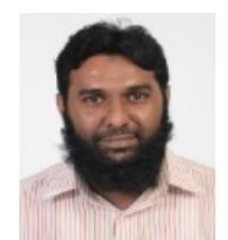

Muhammad Salman Bashir completed his M.Sc. degree in Computer Science from PUCIT, Lahore, and the M.S. degree in Computer Science from COMSATS University, Lahore, Pakistan. He is currently pursuing the Ph.D. degree with the Department of Computer Science and Engineering, University of Engineering and Technology (UET), Lahore. Currently he is working as Assistant Professor with the Department of Computer Science and Information Technology, Virtual University of Pakistan. His research interests include HCI, usability evaluation, software processes, and software requirements engineering.

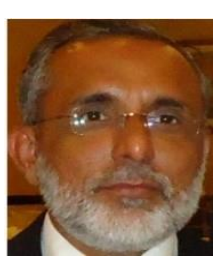

Muhammad Anwaar Saeed joined Virtual University (VU) of Pakistan in April 2006 and is currently working as an Assistant Professor and Head of Computer Science Department. He has obtained his $\mathrm{PhD}$ Degree in Computer Science from National College of Business Administration \& Economics (NCBA\&E), Lahore, Pakistan. His area of research is key generation for data encryption and information security. $\mathrm{He}$ is also interested in Quantum Computing especially encryption mechanisms used in this field. He is also the author of book "Framework for Self Organizing Encryption in Ubiquitous Environment", published by VDM Verlag in 2010. He has published many research papers on his area of interest. Before joining VU, he has ample experience of both software development and network management. 
How to cite this paper: Ahmed Iqbal, Shabib Aftab, Israr Ullah, Muhammad Salman Bashir, Muhammad Anwaar Saeed, "A Feature Selection based Ensemble Classification Framework for Software Defect Prediction", International Journal of Modern Education and Computer Science(IJMECS), Vol.11, No.9, pp. 54-64, 2019.DOI: 10.5815/ijmecs.2019.09.06 